\title{
Mutagénesis ambiental y el uso de biomarcadores para predecir el riesgo de cáncer
}

\author{
Patricia Cuenca ${ }^{1,2}$ * \& Vanessa Ramírez ${ }^{1,3}$ \\ 1 Instituto de Investigaciones en Salud (INISA). Universidad de Costa Rica, 2060, San José, Costa Rica. \\ 2 Escuela de Biología. Universidad de Costa Rica, 2060, San José, Costa Rica. \\ 3 Escuela de Nutrición. Universidad de Costa Rica, 2060, San José, Costa Rica. \\ * Fax (506) 207 5130; correo electrónico: pcuenca@cariari.ucr.ac.cr
}

Recibido 22-VII-2004. Corregido 12-VIII-2004. Aceptado 31-VIII-2004.

\begin{abstract}
Environmental mutagenesis and use of biomarkers in cancer risk prediction. The field of environmental mutagenesis or toxicology genetics aims to study the genetic damage that leads to mutations produced by physical, chemical and biological agents, to identify these agents and analyze their interactions and ways of action. There are enough experimental and epidemiological evidences implicating mutations in oncogenes, tumor suppressor genes and DNA repair genes as determinants in the onset and progression of the neoplastic process. A valuable tool in public and occupational health is the monitoring of populations exposed to potentially hazardous agents. The objective is to protect the health and quality of life of high risk groups on account of the nature of the agents of exposure. Monitoring of genotoxic effects in exposed populations as well as the analysis of susceptibility polymorphism are visualized as key tools in the realm of future public and occupational health in order to prevent the occurrence of environmental and specially occupational origin of tumors. This paper reviews the main concepts concerning this issue and refers to studies on the subject in Costa Rica. Rev. Biol. Trop. 52(3): 585-590. Epub 2004 Dic 15.
\end{abstract}

Key words: Costa Rica, environmental mutagenesis, pesticides, population monitoring, toxicology.

Palabras clave: Costa Rica, mutagénesis ambiental, plaguicidas, monitoreo poblacional, toxicología.

Se puede considerar que la mutagénesis ambiental se inició en 1928 con los estudios publicados por Muller, realizados en Drosophila melanogaster. Muller demostró que los rayos X causan daño al ADN e inducen mutaciones en las células germinales. Desde ese momento se abrió la posibilidad de crear organismos mutantes y estudiar las mutaciones en condiciones experimentales controladas. Dieciocho años después Auerbach y Robson describieron al gas mostaza, como el primer mutágeno de naturaleza química. Esta sustancia, es un poderoso agente alquilante bifuncional que fue usado extensivamente en la primera guerra mundial entre 1914-1919, en Etiopía en 1936 y en la guerra Irán-Iraq entre 1980-1988. Se dice que es radio-mimético debido a que produce los mismos efectos que la exposición excesiva a los rayos X: quemaduras en la piel, pérdida del cabello, inmunosupresión, inducción de aberraciones cromosómicas y cáncer (Venitt y Phillips 1995). Ames en 1979 introdujo el ensayo de mutagenicidad en Salmonella typhimurium, e identificó varios contaminantes químicos capaces de causar mutaciones en bacterias y cáncer en mamíferos (Ames 1979). En 1982 se demostró que una mutación puntual es capaz de conferir capacidad transformadora al oncogén T24, promoviendo el desarrollo de cáncer de vejiga (Reddy et al. 1982). Ahora se sabe, que además de los agentes físicos y químicos también los agentes biológicos como virus, bacterias y parásitos generan mutágenos 
cuando producen infecciones crónicas (Venitt y Phillips 1995). Entre los egipcios, por ejemplo, la infección con el parásito Schistosoma es el primer factor de riesgo para cáncer de vejiga (Au 2001).

La mutagénesis ambiental o genética toxicológica es una disciplina que tiene como objetivo estudiar las mutaciones inducidas por agentes físicos, químicos y biológicos, identificar estos agentes, analizar sus interacciones y mecanismos de acción (Creus 2001). Los estudios de toxicología genética enfocan su atención en niveles de exposición que no se consideran tóxicos, porque no causan efectos inmediatos identificables fácilmente, pero que tienen su efecto sobre el ADN. En 1983 la Comisión internacional para la protección contra mutágenos y carcinógenos ambientales estableció una definición que limita el uso del término genotóxico a aquellos agentes que tienen afinidad para interaccionar con el ADN. Entonces los agentes genotóxicos se definen, como aquellos que causan daño al material genético a dosis subtóxicas (ICPEMC 1983).

Con respecto a los métodos usados por la toxicología genética, existe una amplia gama dependiendo de los objetivos que se deseen alcanzar. Como el objetivo, en la mayoría de los casos, es determinar si un agente en particular es capaz de inducir daño al ADN, se han desarrollado modelos de estudio para análisis de modificaciones en el ADN (como aductos) y de las mutaciones antes y después de la exposición al agente de interés. Es así como existen modelos con bacterias, algas, células eucarióticas, mamíferos y mamíferos modificados por ingeniería genética. Los ensayos pueden ser diseñados in vitro o in vivo. También pueden llevarse a cabo ensayos específicos para las células germinales, y de esta manera observar el efecto sobre la reproducción (Phillips y Venitt 1995).

Hasta el presente se han acumulado suficientes evidencias experimentales y epidemiológicas que demuestran que el daño genético produce mutaciones. Además, el proceso cancerígeno se inicia y se favorece por la presencia de mutaciones en los oncogenes, los genes supresores de tumores y los que codifican para los sistemas de reparación del ADN (Au 2001, Bonassi y Au 2002).

Se ha demostrado en roedores que aquellos agentes que causan mutaciones somáticas también causan mutaciones en sus gametos; no obstante ha sido difícil demostrar que las células reproductoras humanas se ven dañadas de igual manera que las células somáticas con el efecto de alguna exposición, y que por esta causa se ve aumentada la incidencia de enfermedades hereditarias en la población (Venitt y Phillips 1995, Creus 2001). Sin embargo, el aumento en la incidencia de tumores que se viene observando en niños muy pequeños hacen pensar que la exposición paterna o materna a cancerígenos podría ser, en estos casos, el factor que desencadena el desarrollo de un tumor (Fabia y Thuy 1974, Holly et al. 1992, Winn et al. 1992, Sharpe et al. 1995).

El ser humano introduce continuamente al ambiente miles de nuevas sustancias químicas para diversos usos, producción agrícola, industria química, producción de energía, medicina, etc. El potencial tóxico real de estas sustancias sobre los seres humanos se estudia más o menos a profundidad cuando se trata de medicamentos -otras sustancias de uso industrial y agrícola no son analizadas para efectos a largo plazo, debido a que las legislaciones vigentes más exigentes en la mayoría de los países, demandan que las autoridades demuestren el potencial tóxico de una sustancia nueva en un plazo muy corto (unos 90 días), para dar los permisos de comercialización (Landis y Yu 1999). Lo lógico sería que los fabricantes prueben la inocuidad de ellas, antes de sacarlas a la venta- esto ha provocado que grupos de personas expuestas, sobre todo por razones laborales, sufran las consecuencias negativas de dichas exposiciones. Esta realidad ha promovido que la Agencia Internacional para la Investigación en Cáncer (IARC) clasifique, no solamente las sustancias, agentes físicos y biológicos, sino también las ocupaciones según su grado de peligrosidad, como factor de riesgo de desarrollar un cáncer (Anónimo 1994). Es prioritario estudiar el efecto genotóxico de 
todas las sustancias que son liberadas al ambiente, con el fin de cuidar la salud de las poblaciones humanas y del ecosistema. Si las presiones económicas de la sociedad moderna están impidiendo que existan controles adecuados de las sustancias antes de comercializarlas, entonces se debe suplir el vacío de información mediante investigaciones adecuadas. Un entorno saludable y libre de tóxicos es una de las formas que permitiría prevenir y disminuir el riesgo de desarrollar tumores y otras enfermedades degenerativas.

El monitoreo de grupos de población expuestos a agentes potencialmente dañinos para el ser humano es una herramienta valiosa en salud pública y ocupacional. Tiene como objetivo preservar la salud y la calidad de vida en aquellos grupos de trabajadores que son de alto riesgo por la naturaleza de las sustancias a que están expuestos. Es posible utilizar diferentes marcadores y técnicas de monitoreo, desde que el tóxico entra al cuerpo, cuando interacciona con su sitio de acción, en los diferentes momentos de su metabolismo, y hasta que causa enfermedad o la muerte (Anónimo 1999). En relación a los trabajadores agrícolas, por ejemplo, se hacen determinaciones de los niveles de acetilcolinesterasa en suero y eritrocitos, como marcadores de exposición a organofosforados y carbamatos, para protegerlos de los efectos nocivos sobre el sistema nervioso que produce la exposición a éstas sustancias.

Si el objetivo es hacer monitoreo de personas expuestas para evaluar daño genético en las células somáticas, se pueden aplicar diferentes ensayos: (1) los micronúcleos, tanto en linfocitos, como en células epiteliales descamadas de las mucosas, (2) las aberraciones cromosómicas, en sus versiones más clásicas como la búsqueda de lagunas, fracturas y cromosomas dicéntricos en cultivos convencionales, así como intercambio de cromátidas hermanas o (3) en la aplicación de métodos de citogenética molecular para buscar inversiones, translocaciones, o para identificar el origen cromosómico de los micronúcleos. Todos estos se consideran marcadores de efecto temprano, lo cual significa que permiten detectar un nivel de daño que todavía es reversible (Bonassi y Au 2002). Se pudo demostrar en estudios prospectivos realizados en países europeos, que la mayor frecuencia de aberraciones cromosómicas en las personas expuestas es un buen predictor del aumento del riesgo para cáncer (Hagmar et al. 1998).

La experiencia obtenida con el óxido de etileno, permite concluir que las aberraciones cromosómicas se pueden usar como predictores y para prevenir enfermedad al igual que se hace con los ensayos de acetilcolinesterasa y la exposición a los carbamatos y organofosforados. Se observó que los trabajadores expuestos a oxido de etileno presentaban altas frecuencias de aberraciones cromosómicas y que, cuando se interrumpía la exposición el metabolismo era capaz de reparar el daño por lo que disminuía considerablemente la frecuencia de aberraciones cromosómicas después de un período de descanso. Estas observaciones permitieron a la IARC (Anónimo 1994) clasificar a esta sustancia como carcinógeno. Actualmente se considera que las aberraciones cromosómicas son muy útiles, porque después de monitorear las personas expuestas, se les podría dar períodos mayores de descanso a aquellas más susceptibles de sufrir daño genético a esa exposición determinada, o se les podría cambiar de actividad dentro de sus trabajos. Con las metodologías citogenéticas, moleculares y bioquímicas que existen actualmente, es posible detectar cambios o alteraciones que servirían como señal de alarma y entonces tomar las medidas oportunas para minimizar el riesgo para la salud (Bonassi y Au 2002).

Es importante destacar que el potencial genotóxico de una sustancia depende de la variabilidad individual. Las características genéticas en el metabolismo de los xenobióticos, así como también de la capacidad para realizar la reparación del $\mathrm{ADN}$ en el momento oportuno, y la capacidad que tengan las células para entrar en apoptosis y autodestruirse cuando el daño ha sido excesivo, son diferentes en cada persona. Como resultado de esto, la tendencia 
actual consiste en combinar los estudios de monitoreo del daño genético con estudios de polimorfismos en diferentes enzimas que participan de esos procesos metabólicos; ya que se han logrado identificar polimorfismos que confieren una mayor o menor susceptibilidad a determinada exposición (Au et al. 1999, Vineis et al. 1999, Bonassi y Au 2002).

El estudio de los marcadores biológicos en asociación con el riesgo de sufrir alguna enfermedad degenerativa multifactorial es la disciplina que ahora se conoce como epidemiología molecular (Schulte y Perera 1993).

En Costa Rica se estima que el 18\% de la población trabaja en la industria y un $20 \%$ en labores agrícolas. Un estudio publicado en 1997 informa que el $60 \%$ de las industrias utilizan alguna sustancia con potencial cancerígeno. Los trabajadores de la industria están expuestos al menos a 30 sustancias clasificadas por la IARC como del tipo A1 (carcinógeno humano comprobado), A2 (carcinógeno humano potencial) y A3 (carcinógeno animal comprobado) (Morales 1997). Hasta el momento no se ha realizado ningún tipo de estudio sobre el efecto genotóxico de las exposiciones a dichas sustancias en los trabajadores de la industria, a pesar de que, se estima que aplicando diferentes estrategias de protección y prevención podrían evitarse entre 300 y 900 cánceres por año (Morales 1997).

La población agrícola de Costa Rica por su parte, está sujeta a grandes exposiciones de plaguicidas, mayores que lo usual en los países templados, ya que el clima permite la producción prácticamente todo el año. El efecto de los plaguicidas sobre la salud de la población en general y los trabajadores en particular, ha sido y es estudiado por el Programa de Plaguicidas, actualmente IRET, de la Universidad Nacional Autónoma de Costa Rica, en diferentes aspectos como efecto neurotóxico, incidencia de tumores y otros.

En Costa Rica se han hecho pocos estudios sobre efecto genotóxico, en los ochenta la médico Isabel Castro y la bióloga Luisa Valle investigaron el daño cromosómico causado por el virus de la inmunodeficiencia humana (VIH) (Castro-Volio y Valle-Bourrette 2002). Un grupo de investigadores de la Universidad de Texas estudiaron el daño genético en trabajadores bananeros que habían quedado estériles por exposición al DBCP (Au et al. 1999). A mediados de los noventa se presentó la oportunidad de concursar por fondos donados por la Agencia Danesa de Cooperación, administrados por la Organización Panamericana de la Salud, para realizar un estudio sobre el efecto genotóxico de la exposición ocupacional en mujeres que trabajan empacando el banano. Se contó también con el apoyo del Departamento de Sustancias Tóxicas y Medicina del Trabajo del Ministerio de Salud, así como de la Vicerrectoría de Investigación de la Universidad de Costa Rica, del CONICIT y del Servicio Alemán de Intercambio Académico (DAAD). De esta manera, se hizo por primera vez en el país una investigación de este tipo. Se trabajó con marcadores de efecto temprano, como micronúcleos en linfocitos, en mucosa bucal, aberraciones cromosómicas y electroforesis de células únicas. Se logró concluir que la exposición directa a los agentes utilizados en la poscosecha, imazalil, tiabendazole, así como el insecticida clorpirifos (usado para impregnar las bolsas que protegen la fruta en el campo), y/o la exposición indirecta a los plaguicidas usados en los campos de cultivo de banano son genotóxicos, ya que aumentan la frecuencia de aberraciones cromosómicas y el daño en la hebra sencilla del ADN (Ramírez y Cuenca 2001, 2002, Castro et al. 2004, Cuenca y Ramírez 2004). También, y gracias al monitoreo paralelo de los niveles de colinesterasa sérica y eritrocítica en el grupo de expuestas y en el grupo de no expuestas, se observó que, no solamente la población de mujeres que viven y trabajan en una plantación bananera, sino que también aquellas que viven en la ciudad de Guápiles están expuestas a niveles altos de organofosforados y carbamatos, ya que tienen porcentajes de inhibición de éstas enzimas mucho mayores que la población general (Cuenca et al. 1997). 
Para averiguar la importancia relativa que el tiabendazol, imazalil y el clorpirifos pueden tener como agentes genotóxicos, se realizaron evaluaciones de cada plaguicida en forma independiente, con este objetivo se diseñaron ensayos in vitro con células humanas para aberraciones cromosómicas y electroforesis de células únicas (Vindas et al. 2004).

El conocimiento actual acumulado, proveniente de estudios epidemiológicos y experimentales, así como la caracterización de los marcadores biológicos, permite vislumbrar al monitoreo del efecto genotóxico en poblaciones expuestas, en conjunto con el análisis simultáneo de polimorfismos de susceptibilidad, como herramientas clave en la salud pública y ocupacional del futuro, con el fin de prevenir la ocurrencia de tumores de origen ambiental y especialmente laboral. Por otro lado los estudios de monitoreo son útiles para documentar efectos a largo plazo de sustancias que se sacan al mercado sin conocerse con exactitud ese tipo de efectos.

\section{RESUMEN}

La mutagénesis ambiental o genética toxicológica es una disciplina que tiene como objetivo estudiar el daño genético que conduce a mutaciones, producido por agentes físicos, químicos y biológicos, identificar estos agentes, analizar sus interacciones y mecanismos de acción. Existen suficientes evidencias experimentales y epidemiológicas que demuestran que el proceso cancerígeno se inicia y se favorece por la presencia de mutaciones en los oncogenes, los genes supresores de tumores y los que codifican para los sistemas de reparación del ADN. Una herramienta valiosa en salud pública y ocupacional es el monitoreo de grupos de población expuestos a agentes potencialmente dañinos para el ser humano. Tiene como objetivo preservar la salud y la calidad de vida en aquellos grupos que son de alto riesgo por la naturaleza de las sustancias a que están expuestos. Se vislumbra al monitoreo del efecto genotóxico en poblaciones expuestas, en conjunto con el análisis de polimorfismos de susceptibilidad, como herramientas clave en la salud pública y ocupacional del futuro, para prevenir la ocurrencia de tumores de origen ambiental y especialmente laboral. En este trabajo se revisan los principales conceptos sobre este tema y se hace referencia a los trabajos realizados sobre exposición laboral a potenciales cancerígenos y a la que existe en el monitoreo de poblaciones expuestas en Costa Rica.

\section{REFERENCIAS}

Ames, B.N. 1979. Identifying environmental chemicals causing mutations and cancer. Science 204: 587-593.

Anónimo. 1994. Occupational cancer in developing countries. IARC Scientific. Publications $\mathrm{N}^{\circ} 129$. Lyon. $191 \mathrm{p}$.

Anónimo. 1999. The use of short- and medium-term test for carcinogens and data on genetic effects in carcinogenic hazard evaluation. IARC Scientific Publications $\mathrm{N}^{\circ} 146$. Lyon. $536 \mathrm{p}$.

Au, W.W., C.H. Sierra-Torres, N. Cajas-Salazar, B.K. Shipp \& M.S. Legator. 1999. Cytogenetic effects from exposure to mixed pesticides and the influence from genetic susceptibility. Environ. Health Perspect. 104: 579-583.

Au, W.W. 2001. Life style factors and acquired susceptibility to environmental disease. Int. J. Hyg. Environ. Health. 204: 17-22.

Bonassi, S. \& W.W. Au. 2002. Biomarkers in molecular epidemiology studies for health risk prediction. Mutat. Res. 511: 73-86.

Castro-Volio, I. \& L. Valle-Bourrette. 2002. Chromosomal defects in 34 male homosexuals, half of them with HIV antibodies. Rev. Biol. Trop. 50: 347-353.

Castro, R., V. Ramírez \& P. Cuenca. 2004. Análisis de micronúcleos y otras anormalidades nucleares en el epitelio oral de mujeres expuestas ocupacionalmente a plaguicidas. Rev. Biol. Trop. 52: 207-217.

Creus, A. 2001. Genotoxicidad, mutagénesis y carcinogénesis, pp 17-162. In C. Paz-y-Miño, A. Creus, O. Cabré \& P. Leone (eds.). Genética toxicológica y carcinogénesis. Laboratorio de Genética Molecular y Citogenética Humana. Pontificia Universidad Católica del Ecuador, Quito.

Cuenca, P., V. Ramírez, R. Castro \& K. Schosinsky. 1997. Efecto genotóxico de los plaguicidas en una población costarricense expuesta ocupacionalmente. Informe final PLAGSALUD/OPS. San José. 47 p.

Cuenca, P. \& V. Ramírez. 2004. Aberraciones cromosómicas en trabajadoras expuestas ocupacionalmente a plaguicidas. Rev. Biol. Trop. 52: 219-224.

Fabia, J. \& T. Thuy. 1974. Occupation of father at time of birth of children dying of malignant diseases. Br. J. Prev. Soc. Med. 28: 98-100.

Hagmar, L., S. Bonassi, U. Stromberg, A. Brogger, L.E. Knudsen, H. Norppa, C. Reuterwall \& The European Study Group on Cytogenetic Biomarkers and Health. 1998. Chromosomal aberrations in lymphocytes 
predict human cancer: A report from the European Study Group on Cytogenetic Biomarkers and Health (ESCH). Cancer Res. 58: 4117-4121.

Holly, A., D. Aston \& A.D. Khristiansen. 1992. Ewing's bone sarcoma, paternal occupational exposure and other factors. A. J. Epidemiol 135: 122-129.

ICPEMC. 1983. Screening strategy for chemicals that are potential germ-cell mutagens in mammals. Mutat. Res. 144: 117-177.

Landis, W.G. \& M. Yu. 1999. Environmental toxicology. Impacts of chemical upon ecological systems. Lewis. Nueva York. 390 p.

Morales, R. A. 1997. Sustancias químicas cancerígenas en el sector industrial de Costa Rica: el uso de registros como herramientas de Salud Pública; Rev. Costarric. Salud Pública 6: 11-19.

Phillips, D.H. \& S Venitt (eds.). 1995. Environmental Mutagenesis. Bios Scientific. London. 403 p.

Ramírez, V. \& P. Cuenca. 2001. Micronuclei frecuency in lymphocytes of individuals occupationally exposed to pesticides. Rev. Biol. Trop 49: 1-8.

Ramírez V. \& P. Cuenca. 2002. Daño del ADN en trabajadoras bananeras expuestas a plaguicidas en Limón, Costa Rica. Rev. Biol. Trop. 50: 507-518.

Reddy, E.P., R.K. Reynolds, E.Santos \& M. Barbacid. 1982. A point mutation is responsible for the acquisition of transforming properties by the T24 human bladder carcinoma oncogene. Nature 300: 149-152.

Schulte, P.A. \& F.P. Perera. 1993. Molecular epidemiology. Principles and practices. Academic. Nueva York. 588 p.

Sharpe, C.R, E. L. Franco, B. de Camargo, L.F. Lopes, J.H. Barreto, R.R. Johnsson, M.A. Mauad. 1995. Parental exposures to pesticidas and risk of Wilms' tumor in Brazil. Am. J. Epidemiol. 141: 210-217.

Venitt, A \& D. Phillips. 1995. The importance of environmental mutagens in human carcinogenesis and germline mutation. pp 1-17. In D.H. Phillips \& S. Venitt (eds.). Environmental mutagenesis. Bios Scientific, Londres.

Vindas, R., F. Ortiz, V. Ramírez \& P. Cuenca. 2004. Genotoxicidad de tres plaguicidas utilizados en la actividad bananera de Costa Rica. Rev. Biol. Trop. 52: 197-205.

Vineis, P., N. Malats \& P. Boffetta. 1999. Why study metabolic susceptibility to cancer? pp. 1-4 In P. Vineis, N. Malats, M. Lang, A. d'Errico, N. Caporaso, J. Cuzick \& P. Boffetta. Metabolic Polymorphisms and Susceptibility to cancer. IARC Scientific Publications $\mathrm{N}^{\circ} 148$. Lyon.

Winn, D., L. Robinson, J. Mulvihill, A. Daigle \& J. Fraumeni. 1992. A case-control study of the aetiology of Ewing's sarcoma.Cancer. Epidemiol. Biomarkers Prev. 1: 525-532. 